\title{
Hybrid repair of Kommerell diverticulum
}

\author{
Jahanzaib Idrees, MD, ${ }^{a}$ Suresh Keshavamurthy, MD, ${ }^{a}$ Sreekumar Subramanian, MD, ${ }^{a}$ Daniel G. Clair, MD, \\ Lars G. Svensson, MD, PhD, ${ }^{\mathrm{a}}$ and Eric E. Roselli, $\mathrm{MD}^{\mathrm{a}}$
}

Objective: Kommerell diverticulum carries the risk of rupture or dissection if left untreated. Various methods of repair have been described, and options have recently expanded to include the hybrid approach. This study describes hybrid repair techniques for Kommerell diverticulum and assesses outcomes.

\begin{abstract}
Methods: Between 2005 and 2010, a total of 10 patients underwent hybrid repair of Kommerell diverticulum (3 right-sided arches) by elephant trunk with endovascular completion $(n=4)$, frozen elephant trunk $(n=3)$, or stent grafting with cervical debranching $(\mathrm{n}=3)$. Mean age at procedure was $57 \pm 25.5$ years. Subclavian artery revascularization was performed preoperatively $(n=4)$, intraoperatively $(n=3)$, or postoperatively $(n=3)$, either as carotid-to-subclavian bypass $(\mathrm{n}=7 ; \mathrm{n}=3$ bilateral $)$ or originating from the ascending aorta $(\mathrm{n}=3)$. Data were obtained from the prospectively collected database and chart review.
\end{abstract}

Results: There was no in-hospital mortality, nor were there any cases of respiratory or renal failure. There were no cases of paraplegia, but there was 1 stroke. Two patients had type 2 endoleaks develop; 1 required subclavian coil embolization. Mean hospital stay was $8.73 \pm 4$ days. After repair, there were no ruptures, no significant growth, and all patients remain free of symptoms.

Conclusions: Hybrid repair is a safe and effective surgical treatment option for Kommerell diverticulum. Selection of the specific type of intervention is based on patient anatomy and comorbid conditions. ( $\mathrm{J}$ Thorac Cardiovasc Surg 2014;147:973-6)

Kommerell diverticulum is a rare congenital anomaly described as aneurysmal aortic dilatation at the origin of aberrant subclavian artery. It is usually asymptomatic but carries a $19 \%$ to $53 \%$ risk of rupture or dissection. It may present with symptoms of tracheal and esophageal compression, and this occurs more frequently when it is associated with a right-sided aortic arch. ${ }^{1-3}$

Various surgical techniques have been described for repair of Kommerell diverticulum. Traditionally, the mainstay of treatment has been open repair under conditions of deep hypothermic arrest or partial left heart bypass with resection of the aneurysmal segment and replacement of the involved segment of aorta with an interposition graft. ${ }^{2-4}$ During the past decade, surgical options have expanded to include endovascular and hybrid approaches as suitable operative strategies to manage complex aortic disease. The study objectives were to describe hybrid repair techniques for Kommerell diverticulum in adults and to assess their outcomes.

\footnotetext{
From the Departments of Thoracic and Cardiovascular Surgery and Vascular Surgery, ${ }^{\mathrm{b}}$ Heart and Vascular Institute, Cleveland Clinic, Cleveland, Ohio. Disclosures: Authors have nothing to disclose with regard to commercial support. Presented at Aortic Surgery Symposium 2012, New York, NY, April 26-27, 2012. Received for publication Sept 14, 2012; revisions received Feb 12, 2013; accepted for publication Feb 27, 2013; available ahead of print March 26, 2013.

Address for reprints: Eric E. Roselli, MD, Department of Thoracic and Cardiovascu-

lar Surgery, Cleveland Clinic, 9500 Euclid Ave, Desk J4-1, Cleveland, OH 44195-5108 (E-mail: roselle@ccf.org).

$0022-5223 / \$ 36.00$

Copyright (c) 2014 by The American Association for Thoracic Surgery http://dx.doi.org/10.1016/j.jtcvs.2013.02.063
}

\section{MATERIALS AND METHODS}

From August 2005 to October 2010, a total of 10 adults underwent repair of Kommerell aortic diverticulum with hybrid techniques at Cleveland Clinic. The mean age at the time of operation was $57 \pm 25$ years. Seven patients had aberrant origin of the right subclavian artery, and 3 had right-sided aortic arch with retroesophageal left subclavian artery. Four patients were seen with tracheoesophageal compression and had symptoms of chest or back pain at the time of the index operation, and 5 patients had associated dissection. The remaining had large aneurysms found incidentally. Table 1 summarizes preoperative patient characteristics. Data were obtained from retrospective chart review, augmented with the social security death index.

\section{Repair Techniques}

Patients underwent repair with 1 of 3 hybrid strategies: (1) 2-stage elephant trunk repair with endovascular completion $(n=4),(2)$ single-stage frozen elephant trunk repair $(n=3)$, or (3) thoracic endovascular aortic repair with cervical debranching $(n=3)$. One patient was treated on an emergency basis for acute dissection with a frozen elephant trunk operation; the others were treated electively. All patients underwent subclavian artery revascularization either preoperatively $(n=4)$, intraoperatively $(\mathrm{n}=3)$, or postoperatively $(\mathrm{n}=3)$. The revascularization procedures included carotid-to-subclavian bypass $(\mathrm{n}=7)$ or ascending to subclavian bypass $(\mathrm{n}=3)$. Subclavian arteries were revascularized bilaterally in 4 patients.

Elephant trunk with endovascular completion. This approach was chosen for patients with extensive disease involving the more distal thoracic aorta. All 4 of these patients had chronic aortic dissection. The first stage was the conventional first-stage elephant trunk operation performed through a median sternotomy under hypothermic circulatory arrest with selective antegrade brain perfusion. ${ }^{5,6}$ Three patients had total arch replacement with reconstruction of the supra-aortic branches. The other patient had previously undergone stent grafting of an acute type B dissection complicated by malperfusion. On follow-up, he was found to have a persistent endoleak related to Kommerell diverticulum and aberrant 
TABLE 1. Preprocedural patient characteristics

\begin{tabular}{lc}
\hline Age $(y$, mean and range) & $57(31-82)$ \\
Male & $6(60 \%)$ \\
Maximum aneurysmal diameter $(\mathrm{cm}$, mean $\pm \mathrm{SD})$ & $4.7 \pm 0.85$ \\
Right-sided aortic arch & $3(30 \%)$ \\
Aberrant right subclavian artery & $7(70 \%)$ \\
Aberrant left subclavian artery & $3(30 \%)$ \\
Aortic dissection & $5(50 \%)$ \\
Proximal aortic aneurysm & $4(40 \%)$ \\
Symptomatic & $4(40 \%)$ \\
Need for concomitant CABG & $3(30 \%)$ \\
\hline
\end{tabular}

Data are number of patients with percentage except as specified. $C A B G$, Coronary artery bypass grafting; $S D$, standard deviation.

subclavian artery. The first-stage elephant trunk was constructed through an arch aortotomy without proximal aortic replacement. Aortoplasty and open fenestration to create a distal descending aortic landing zone were performed in 2 patients with extensive distal dissection. Details of this operation have been previously described.?

The second stage of the elephant trunk was completed with an endovascular stent-graft device by transfemoral delivery under fluoroscopic guidance in all 4 patients. Median interval from stage 1 to endovascular stage 2 completion was 51 days.

Frozen elephant trunk. Three patients underwent hybrid repair of Kommerell diverticulum with the frozen elephant trunk operation. These patients had right-sided aortic arch and retroesophageal left subclavian artery or type 3 complete vascular ring.

The technique of frozen elephant trunk repair has been previously described, but some additional details are relevant in these cases. ${ }^{8}$ Briefly, retrograde access into the ascending aorta was first obtained with a catheter through the groin under fluoroscopic guidance. Mini upper $\mathbf{J}$ sternotomy was then performed. ${ }^{9}$ Under conditions of circulatory arrest with retrograde brain perfusion, the thoracic aorta was incised longitudinally between the common carotid arteries, and a wire was placed antegradely ${ }^{10}$ through the previously placed catheter from the groin. A stent-graft was then deployed antegradely into the descending aorta. In all cases, the left subclavian artery was covered. The proximal part of the stent-graft was then sutured to the native aorta within the arch for fixation and sealing. The longitudinal aortotomy was closed. The vascular ring was divided in all patients by the ligation and transection of the ligamentum arteriosum to release compression of the trachea and esophagus (Figure 1).

\section{Thoracic endovascular stent grafting with cervical de-}

branching. This technique was performed in 3 patients. It was conducted in 2 stages. The first stage included surgical revascularization of the subclavian artery. Bilateral carotid subclavian bypass was performed in 2 patients, and right carotid to aberrant right subclavian bypass was performed in 1 . The second stage included endovascular stent grafting of the distal arch and descending aorta. This was achieved by accessing the ascending aorta through the femoral artery and then deploying the stent-graft in a retrograde fashion under fluoroscopic guidance. Both aberrant right and left subclavian arteries were covered in all patients. Coil embolization of the subclavian artery was also performed at the same time in all 3 patients, to prevent retrograde filling of the excluded aneurysmal sac.

\section{Outcomes and Statististics}

Technical success was defined as the ability to complete the operation with the patient surviving combined with fluoroscopic documentation of complete exclusion of the aneurysmal segment of thoracic aorta including the Kommerell diverticulum without type 1 and 3 endoleaks. Because of
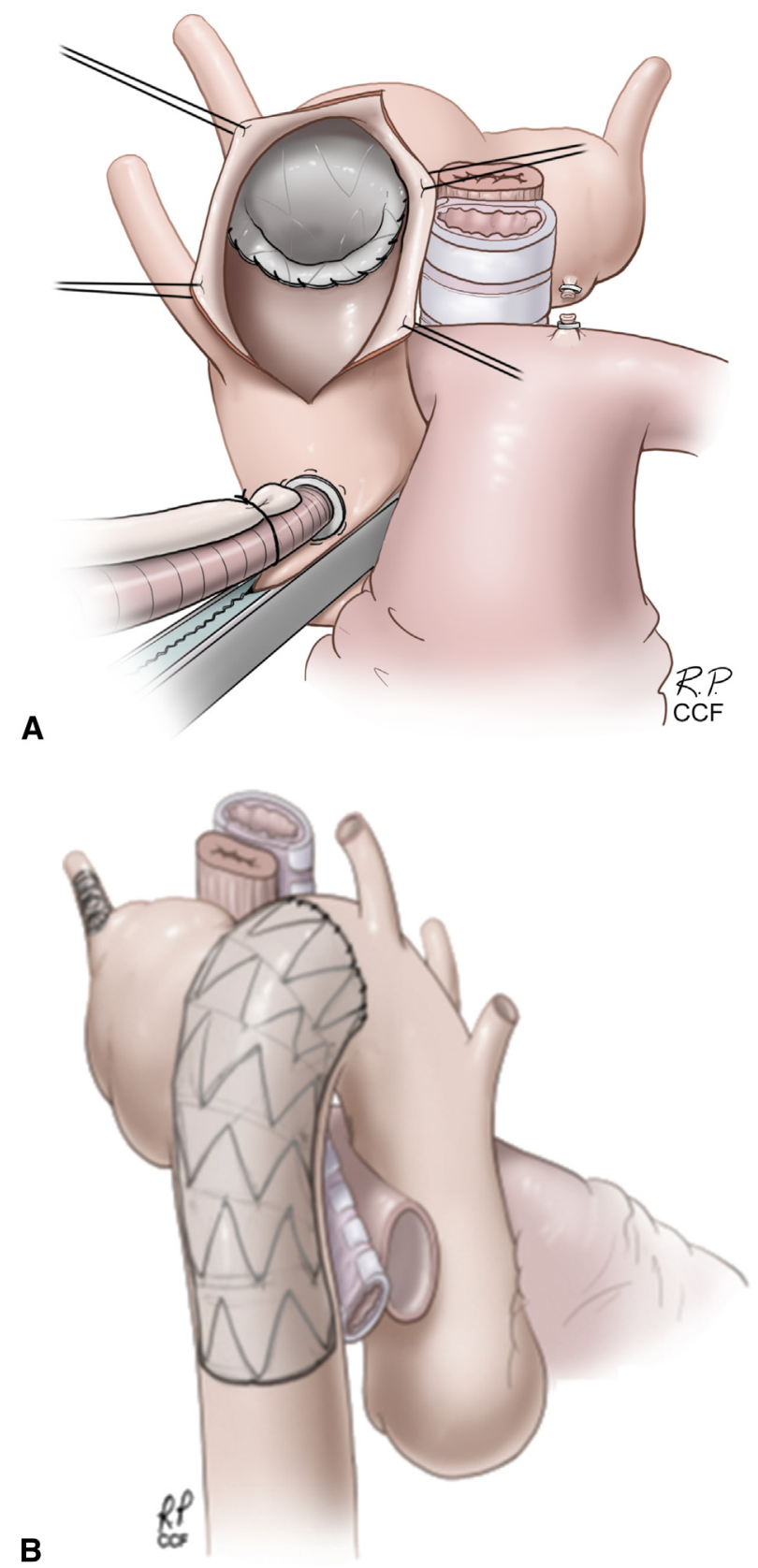

FIGURE 1. Single-stage frozen elephant trunk repair of Kommerell diverticulum. A, Anterior view shows the antegrade stent-graft delivery into the descending aorta through an aortotomy. B, Posterior view shows the stent-graft in place and the coil embolization of the left subclavian artery.

the multistage nature of hybrid repair, reintervention was defined as the need for an extra, unplanned procedure to address complications after the initial repair. Renal failure was defined as the need for hemodialysis, and respiratory failure was defined as the need for reintubation or tracheostomy postoperatively. Follow-up was performed as scheduled outpatient visits, which included imaging by computed tomographic angiography that was analyzed with 3-dimensional reconstruction software (TeraRecon, Inc, San Mateo, Calif) to assess graft patency, device integrity, endoleaks, and aneurysmal morphology. 

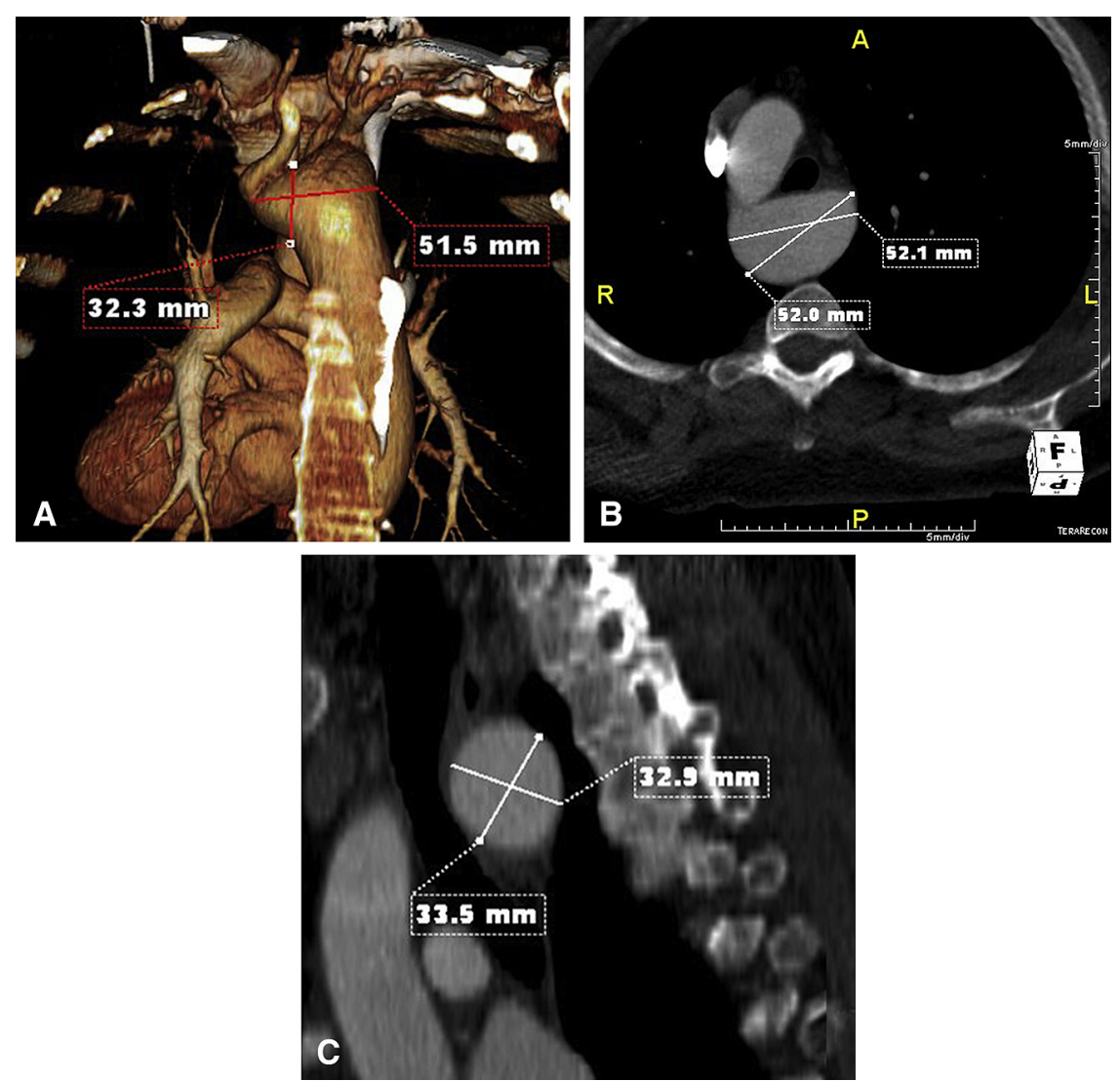

FIGURE 2. Multiplanar volume-rendered reconstruction of computed tomography. A, Volume-rendered image of Kommerell diverticulum shows the measurements in 2 dimensions. B, The diameter as measured cross-sectionally from the opposite aortic wall to the tip of the Kommerell diverticulum. $\mathrm{C}$, The diameter as measured cross-sectionally at the origin of the aberrant subclavian artery from the aortic arch.

Descriptive statistical analyses are used to present variables in the study. Continuous variables are presented as median or mean $\pm \mathrm{SD}$, and categoric variables are presented as percentages.

\section{RESULTS}

\section{Early Outcomes}

Technical success was $100 \%$. There was no in-hospital or 30-day mortality. Mean hospital stay was $8.73 \pm 4$ days. There were no reoperations for bleeding and no cases of respiratory failure, renal failure, or paraplegia. One patient had a posterior circulation embolic stroke after stent grafting with cervical debranching. Symptoms included altered vision, speech difficulty, and facial weakness, all of which resolved during follow-up.

\section{Late Outcomes}

There were no late deaths at a mean follow-up of $1.8 \pm 1.5$ years. One patient later required carotid subclavian bypass for right upper limb ischemia. Among the patients who underwent stent-graft repair of Kommerell diverticulum, 2 required brachial artery thrombectomy and 2 had type 2 endoleak develop, 1 requiring subclavian artery coil embolization later. After repair, there were no cases of significant aortic growth, and all patients are symptom free.

\section{DISCUSSION}

Various treatment options for the rare condition of Kommerell diverticulum have been previously described. Our experience with the hybrid approach demonstrates the safety and feasibility of another option.

Currently, there are no well-established guidelines for the treatment of Kommerell diverticulum, limitations being the rare occurrence of disease and a heterogeneous population with diverse presentation. Nonetheless, staged open surgical repair composed of resection and replacement with a tube graft, followed by subclavian artery revascularization, has been the most commonly described technique, and the outcomes reported are variable. In review of 32 cases by Austin and Wolfe, open surgical treatment was performed in 20 patients with a perioperative mortality of $40 \% .^{11}$ In comparison, Kouchoukos and colleagues ${ }^{4}$ reported no deaths after standard surgical resection in $10 \mathrm{pa}-$ tients; however, they did see 4 late deaths at mean follow-up of 1.5 to 44 months. In our experience with hybrid repair, there was no mortality at a mean follow-up of $1.8 \pm 1.5$ years.

Choice of the specific technique used is based on patient comorbidities, anatomy, and surgeon's preference. Less 
invasive options are preferred as long as the end result is not compromised. Many patients, however, do not have adequate landing zones for fixation of a stent-graft even with adjunctive cervical debranching. In our small experience, only 3 patients had anatomy amendable to this approach, which did not require a chest incision. The 2-stage and single-stage (frozen) hybrid elephant trunk techniques address the issue of a limited proximal landing zone by approaching the arch through a sternotomy with the patient under circulatory arrest. With the frozen elephant trunk technique, the stent-graft is directly sutured into the aortic arch, allowing a much shorter proximal landing zone. With the two-stage elephant trunk technique, the graft is sutured into the arch, creating a longer landing zone. The use of stent-grafts to perform the distal portion of the reconstruction with either of these modified elephant trunk techniques obviates the need for a thoracotomy incision. Another important consideration when choosing repair techniques is the presence of symptoms, because some patients have dysphagia develop as a result of compression from the aneurysmal sac. Sealing off the aneurysm with endovascular placement of graft material will readily relieve pressure within the aneurysmal sac, but that may not be adequate in patients with complete vascular ring. A sternotomy allows access to the heart and the ligamentum arteriosum, which may be divided to release the vascular ring. Other important factors in the decision-making process include the need for concomitant cardiac procedures, in which case a hybrid elephant trunk option works well. If more extensive distal aortic repair is required, a twostaged approach may reduce the risk of spinal cord injury relative to a single-stage repair.

Use of modern imaging with advanced 3-dimensional reconstruction has made it possible to analyze in detail the complex anatomy of Kommerell diverticulum and arch anomalies to guide preoperative planning. The unusual morphology of this entity makes it difficult to describe the dimensions of the involved aortic segments in a standard fashion. Prophylactic repair has been recommended for aneurysmal sizes of 3 to $5 \mathrm{~cm}$ or greater; however, the specific details of where to make these measurements are not always clear. ${ }^{1,2,4}$ Our method of measuring Kommerell diverticulum is presented in Figure 2. Two measurements are used to describe the involved segment of aorta. One is at the level of origin of the aberrant subclavian artery from the arch. In this plane, a diameter of at least $3 \mathrm{~cm}$ is considered an indication to operate. The second is at the cross-sectional diameter from opposite aortic wall to the tip of the Kommerell diverticulum. If the second measurement is at least $5 \mathrm{~cm}$, we usually recommend prophylactic repair.

In conclusion, the options for repair of Kommerell diverticulum may safely include hybrid techniques. Selection of the best treatment for this uncommon pathology depends on a detailed understanding of the patient's anatomy, as derived from 3-dimensional imaging, and recognition of the benefits and limitations of the various repair strategies.

\section{References}

1. Imagawa H, Kadoba K, Taniguchi K, Sawa Y, Takahashi T, Fukushima N, et al. Saccular aneurysm in the right-sided aortic arch: a successfully corrected case. J Vasc Surg. 1997;25:941-9.

2. Cinà CS, Althani H, Pasenau J, Abouzahr L. Kommerell's diverticulum and right-sided aortic arch: a cohort study and review of the literature. J Vasc Surg. 2004;39:131-9.

3. Backer CL, Mavroudis C, Rigsby CK, Holinger LD. Trends in vascular ring surgery. J Thorac Cardiovasc Surg. 2005;129:1338-47.

4. Kouchoukos NT, Masetti P. Aberrant subclavian artery and Kommerell aneurysm: Surgical treatment with a standard approach. J Thorac Cardiovasc Surg. 2007;133:888-92.

5. Svensson LG. Rationale and technique for replacement of the ascending aorta, arch, and distal aorta using a modified elephant trunk procedure. J Card Surg. 1992;7:301-12.

6. Svensson LG, Kim KH, Blackstone EH, Alster JM, McCarthy PM, Greenberg RK, et al. Elephant trunk procedure: newer indications and uses. Ann Thorac Surg. 2004;78:109-16.

7. Roselli EE, Sepulveda E, Pujara AC, Idrees J, Nowicki E. Distal landing zone open fenestration facilitates endovascular elephant trunk completion and false lumen thrombosis. Ann Thorac Surg. 2011;92:2078-84.

8. Lima B, Roselli EE, Soltesz EG, Johnston DR, Pujara AC, Idrees J, et al. Modified and "reverse" frozen elephant trunk repairs for extensive disease and complications after stent grafting. Ann Thorac Surg. 2012;93:103-9; discussion 109.

9. Svensson LG. Minimally invasive surgery with a partial sternotomy "J" approach. Semin Thorac Cardiovasc Surg. 2007;19:299-303.

10. Roselli EE, Soltesz EG, Mastracci T, Svensson LG, Lytle BW. Antegrade delivery of stent grafts to treat complex thoracic aortic disease. Ann Thorac Surg. 2010;539-46.

11. Austin EH, Wolfe WG. Aneurysm of aberrant subclavian artery with a review of the literature. J Vasc Surg. 1985;2:571-7. 